\title{
Chirality-Selective Fabrication of Carbon Nanotube Gas sensor Using Spin-Column Chromatography and Dielectrophoresis
}

\author{
J. Suehiro, M. Fujioka, H. Watanabe, H. Komure, M. Nakano \\ Kyushu University, 744 Motooka Nishi-ku, Fukuoka, 819-0385 Japan \\ suehiro@ees.kyushu-u.ac.jp
}

\begin{abstract}
:
Carbon nanotube (CNT) gas sensors have received considerable attention because of their outstanding properties. The CNT sensor performance, however, has not been optimized because of the coexistence of CNTs with different electronic properties depending on their chirality. This study demonstrates a chirality-selective fabrication method of the CNT gas sensor using spin-column chromatography and dielectrophoresis (DEP). After passing through three spin-columns, pristine CNTs were separated into three fractions, which contained CNTs with different chiralities. The CNTs in each fraction were trapped on the microelectrodes by DEP to fabricate three CNT gas sensors. Comparison of these CNT gas sensor responses to $\mathrm{NO}_{2}$ gas revealed that the sensor response was dependent on the CNT chirality and could be improved by the chirality separation.
\end{abstract}

Key words: carbon nanotube, gas sensor, chirality, spin-column chromatography, dielectrophoresis

\section{Introduction}

Dielectrophoresis (DEP) is an electrokinetic motion of dielectrically polarized materials in non-uniform electric fields. The authors have demonstrated that the DEP manipulation could provide a way to trap carbon nanotubes (CNTs) onto a microelectrode and that the DEP-trapped CNTs could detect ppm-level $\mathrm{NH}_{3}, \mathrm{H}_{2}$ and $\mathrm{NO}_{2}$ gas at room temperature [1].

Considering that the electronic interaction between semiconducting CNTs and target gas molecules is responsible for the CNT gas sensor response [2], separation of semiconducting CNTs from pristine CNTs, which are generally achieved as a mixture of semiconducting and metallic CNTs, might improve the CNT gas sensor sensitivity. Since the electronic structure (metallic or semiconducting) of CNTs is governed by their chirality, a chirality-selective fabrication of CNT gas sensor is essential to improve their performance [3].

In this study, a chirality-selective fabrication method of CNT gas sensor was demonstrated by combining spin-column chromatography and dielectrophoresis. As they both manipulated CNTs in aqueous phase, proposed technique can ensure an efficient and seamless process for CNT sensor fabrication.

\section{Experimental}

Single-walled CNTs synthesized by a highpressure carbon monoxide process (Unidym, $\mathrm{HiPco}, 0.8-1.2 \mathrm{~nm}$ in diameter) were used as pristine CNTs. The CNTs consists of both types of electrical property (metallic and semiconducting). They were dispersed in $2 \mathrm{wt} \%$ sodium dodecyl sulfate (SDS) at $0.3 \mathrm{mg} / \mathrm{ml}$ by ultrasonication for a period of $8 \mathrm{~h}$. Thus prepared CNT suspension was centrifuged at $16000 \mathrm{~g}$ for $15 \mathrm{~h}$ to remove large bundles and impurities. The resulting supernatant was collected as the pristine CNT suspension.

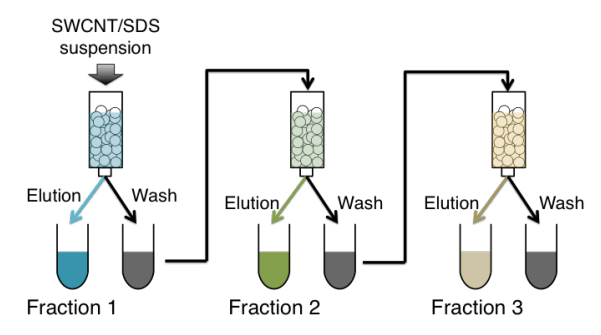

Fig. 1. Chirality separation of CNTs using spin-column chromatography. 
The spin column was filled with dextran-based size-exclusion gel beads $(1 \mathrm{ml}$ of the suspension, GE Healthcare, Sphacryl S200HR). The column was washed and equilibrated by applying a solution of $2 \mathrm{wt} \%$ SDS $(150 \mu \mathrm{l})$ and centrifuged at $750 \mathrm{~g}$ for $60 \mathrm{~s}$. The CNT suspension $(150 \mu \mathrm{l})$ was applied to the column followed by centrifugation at $750 \mathrm{~g}$ for $60 \mathrm{~s}$. While transporting the suspension liquid through the column, CNTs with higher gel affinity were expected to be selectively accumulated on gel beads depending on their chirality. After repeating this procedure 10 times, the CNTs bound onto the gel beads were eluted by injecting $1 \mathrm{wt} \%$ sodiumdeoxycholate (DOC) solution (fraction1). Fractions 2 and 3 were obtained repeating the same procedure by loading the SDS solution passed through the first or second column to the other spin columns (Fig. 1). The optical absorbance spectra of the CNTs of each fraction were measured using a ultraviolet-visible (UV-VIS) spectrophotometer.

In order to fabricate CNT gas sensors, CNTs suspended in each fraction was trapped onto a microelectrode (5 $\mu \mathrm{m}$ gap) under action of positive DEP force generated by ac electric field (100 kHz frequency, $10 \mathrm{~V}$ peak-to-peak value) $[4,5]$. After a desired period of time, the DEP process was stopped, and the suspension liquid was gently dried at room temperature. To investigate the effects of CNT chirality, the gas sensor characteristics were compared between three fractions.

For characterization of gas sensing performance, nitrogen dioxide $\left(\mathrm{NO}_{2}\right)$ gas, which has been widely employed to evaluate CNT gas sensor performance, was used as a target gas. The gas sensor was placed in a chamber (200 ml capacity) made of stainless steel. $\mathrm{NO}_{2}$ gas was generated by using a gas permeater employing dry $\mathrm{N}_{2}$ as a carrier gas. Before exposing the sensor to $\mathrm{NO}_{2}$ gas, the sensor was initialized by UV irradiation (a center wavelength of $365 \mathrm{~nm}$ ) with dry $\mathrm{N}_{2}$ gas flow so that contaminant oxygen molecules adsorbed during the sensor storage and handling in atmospheric air could be removed by photo desorption. The concentration of $\mathrm{NO}_{2}$ was adjusted to $1 \mathrm{ppm}$. The chamber was firstly filled with dry $\mathrm{N}_{2}$ gas and then $\mathrm{NO}_{2}$ gas was introduced at a constant flow rate of $0.5 \mathrm{l} / \mathrm{min}$ using a mass flow controller. The sensor impedance was continuously measured at $100 \mathrm{kHz}$ frequency using the same system as that employed during the DEP sensor fabrication. During the $\mathrm{NO}_{2}$ gas detection experiments, the sensor temperature was maintained at $25^{\circ} \mathrm{C}$ using a ceramic heater and a temperature controller.

\section{Results and Discussion}

As shown in Fig. 2, optical absorption spectra of three fractions had distinctive peaks, which were attributed to different chiral index $(n, m)$ [6]. At this stage, however, proposed method could not realize single chirality separation as demonstrated by Liu et al. using gel chromatography [7]. Fig. 3 compares $\mathrm{NO}_{2}$ responses of three CNT gas sensors, which

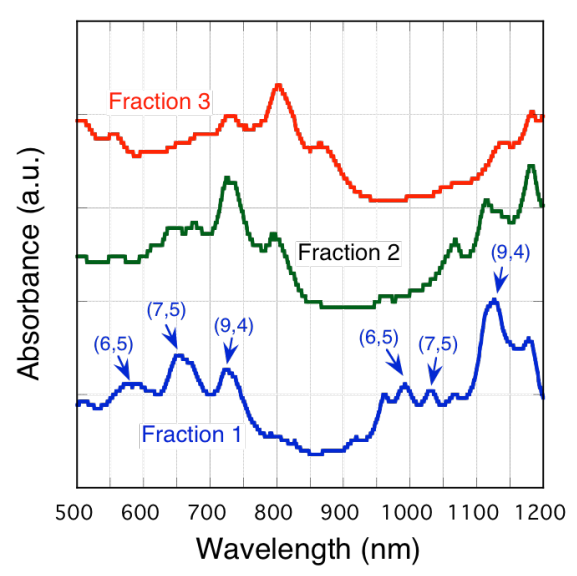

Fig. 2. Optical absorption spectra of the three fractions obtained by spin-column chromatography. Each fraction has distinctive peaks, which are attributed to different chiral index $(n, m)$ of CNTs.

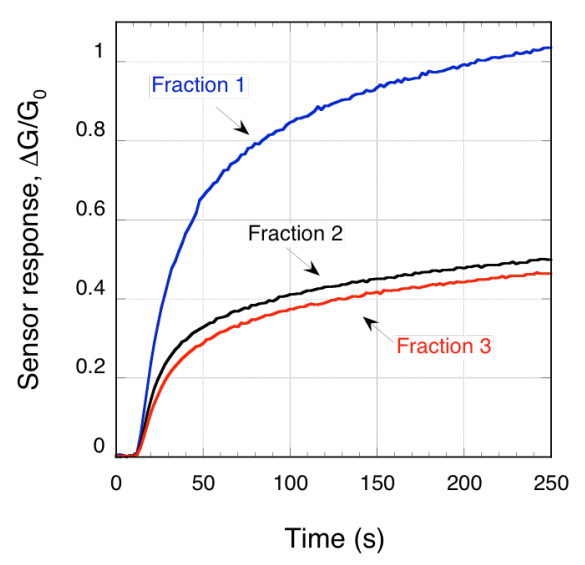

Fig. 3. $\mathrm{NO}_{2}$ responses of three CNT gas sensors, which were fabricated by DEP process using three fractions as CNT suspension. The sensor obtained from fraction 1 showed enhanced response almost two times higher than the other two sensors. 
were fabricated by DEP process using three fractions as CNT suspension. The sensor responses are normalized as $\Delta G / G_{0}$, where $\Delta G$ and $G_{0}$ are the conductance change during $\mathrm{NO}_{2}$ exposure and the initial conductance of CNT sensor, respectively [5]. The sensor obtained from fraction 1 showed enhanced response almost two times higher than the other two sensors, suggesting that chiralities of CNTs in fraction 1 were preferable for $\mathrm{NO}_{2}$ detection. According to Fig. 2, main CNT chirality index of fraction 1 was identified as $(9,4),(7,5)$ and $(6,5)$, which were all classified into semiconducting CNT.

To clarify which chirality of CNT in fraction 1 is most responsible for $\mathrm{NO}_{2}$ detection, additional experiments were conducted using a single spin-column with various loading time of CNT/SDS suspension so that CNTs were enriched depending on the chirality [8]. As depicted in Fig. 4, the CNT sensor response varied with loading time qualitatively in the same manner as the optical absorbance peak at $722 \mathrm{~nm}$, which was attributed to $(9,4)$ chirality index. This result suggests that CNT with $(9,4)$ chirality index might be mainly responsible for $\mathrm{NO}_{2}$ detection.

In order to interpret the chirality dependent CNT sensor response, theoretical analysis of chirality dependent reactivity of $\mathrm{NO}_{2}$ molecules with CNT is necessary for further investigation.

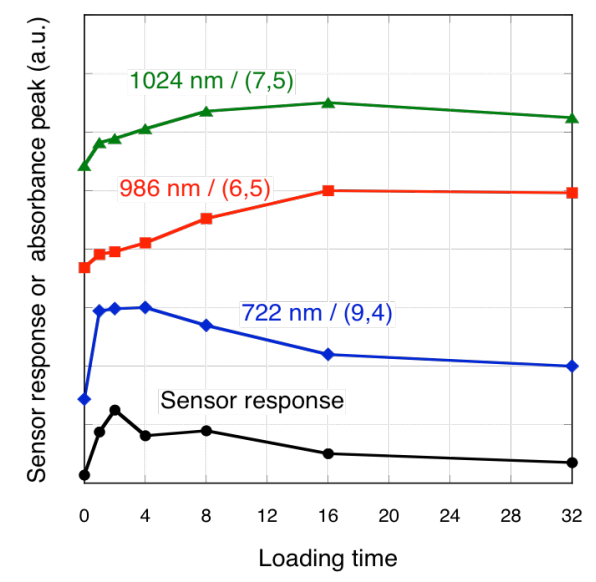

Fig. 4. Variation of optical absorbance peaks and CNT gas sensor response with loading time of CNT/SDS suspension to a single spincolumn. The CNT sensor response varied with loading time qualitatively in the same manner as the optical absorbance peak at $722 \mathrm{~nm}$, which was attributed to $(9,4)$ chirality index.

\section{Conclusion}

We proposed and demonstrated a chiralityselective fabrication method of the CNT gas sensor using spin-column chromatography and dielectrophoresis. The experimental results showed that CNT gas sensor response could be improved by employing CNTs with a specific chiralityas gas sensing transducer using proposed technique.

\section{Acknowledgements}

This work was partly supported by Grants-inAid for Scientific Research from the Japan Society for the Promotion of Science (No. 23360180).

\section{References}

[1] J. Suehiro, Fabrication and Characterization of Nanomaterial-Based Sensors Using Delectrophoresis, Biomicrofluidics, 4, 3430535 (2010); doi: $10.1063 / 1.3430535$

[2] J. Kong, N.R. Franklin, C. Zhou, M.G. Chapline, S. Peng, K. Cho, H. Dai, Nanotube Molecular Wires as Chemical Sensors, Science, 287,622625 (2000); doi: 10.1126/science.287.5453.622

[3] K. Seo,K. A. Park,C. Kim,S. Han, B. Kim, Y. H. Lee, Chirality- and Diameter-Dependent Reactivity of $\mathrm{NO}_{2}$ on Carbon Nanotube Walls, J. Am. Chem. Soc., 127, 15724-15729 (2005); doi: 10.1021/ja052556y

[4] J. Suehiro, G. Zhou, M. Hara, Fabrication of a Carbon Nanotube-Based Gas Sensor Using Dielectrophoresis and its Application for Ammonia Detection by Impedance Spectroscopy, J. Phys. D: Appl. Phys., 36, L109-L114 (2003); doi: 10.1088/0022-3727/36/21/L01

[5] J. Suehiro, G. Zhou, H. Imakiire, W. Ding, M. Hara,Controlled Fabrication of Carbon Nanotube $\mathrm{NO}_{2}$ Gas Sensor Using Dielectrophoretic Impedance Measurement, Sensors and Actuators B: Chemical, 108, 398-403 (2005); doi: 10.1016/j.snb.2004.09.048

[6] S. M. Bachilo, M. S. Strano, C. Kittrell, R. H. Hauge, R. E. Smalley, R. B. Weisman, StructureAssigned Optical Spectra of Single-Walled Carbon Nanotubes, Science, 298, 2361-2366 (2002); doi: 10.1126/science.1078727

[7] H. Liu, D. Nishide, T. Tanaka, H. Kataura, LargeScale Single-Chirality Separation of Single-Wall Carbon Nanotubes by Simple Gel Chromatography, Nature Communications (2011); doi: 10.1038/ncomms 1313

[8] M. Nakano, M. Fujioka, K. Mai, H. Watanabe, Y. Martin, J. Suehiro, Dielectrophoretic Assembly of Semiconducting Carbon Nanotubes Separated and Enriched by Spin Column Chromatography and Its Application to Gas Sensing, Jpn. J. Appl. Phys., 51, $045102 \quad$ (2012); doi: 10.1143/JJAP.51.045102 